\title{
Q-Convergence in graded ditopological texture spaces
}

\author{
RAMAZAN EKMEKÇi AND RIZA ERTÜRK
}

\begin{abstract}
Convergence of graded difilters have been presented and investigated by the authors in [9]. In this paper, using graded Q-dinhd systems defined in [8] the authors define a different convergence type of graded difilters called Q-convergence which has some advantages and some disadvantages in comparison with the convergence defined in [9].
\end{abstract}

\section{INTRODUCTION}

In 1968, C. Chang defined the concept of fuzzy topological space as ordinary subset of the family of all fuzzy subsets of a given set [7]. As a more suitable approach to the idea of fuzzyness, in 1985, Šostak and Kubiak independently redefined fuzzy topology where a fuzzy subset has a degree of openness rather than being open or not $[12,14]$ (for historical developments and basic ideas of the theory of fuzzy topology see [15]).

Ditopological texture spaces were introduced by L. M. Brown as a natural extention of the work on the representation of latticevalued topologies by bitopologies in [10]. The concept of ditopology is more general than general topology, bitopology and fuzzy topology in Chang's sense. An adequate introduction to the theory of texture spaces and ditopological texture spaces may be obtained from $[1-5,17,18]$.

Recently, L. M. Brown and A. Sostak have presented the concept "graded ditopology" on textures as an extention of the concept of ditopology to the case where openness and closedness are given in terms of a priori unrelated grading functions [6]. The concept of graded ditopology is more general than ditopology and fuzzy topology in Šstak's sense. Two sorts of neighborhood structure (graded dineighborhoods and graded Q-dineighborhoods) on graded ditopological texture spaces are presented and investigated in [8]. In [9] graded difilters are introduced and their convergence is studied by means of graded dineighborhoods.

2010 Mathematics Subject Classification. Primary: 54A20; Secondary: 54A05, 06D10.

Key words and phrases. Graded ditopology, convergence, Q-convergence, graded difilter, texture, fuzzy topology. 
The aim of this paper is to introduce an alternative definition of convergence of graded difilters using graded Q-dineighborhoods. There is no relation between graded dineighborhoods and graded Q-dineighborhoods, i.e. they are completely different concepts. So, convergence and Q-convergence of graded difilters are also completely different. Convergence of graded difilters is not defined using graded dineighborhoods but a version of it. Contrary to the concept of convergence, Q-convergence is defined directly by means of Q-dineighborhoods. However, Q-convergence is defined on complemented grounded textures.

\section{Preliminaries}

We recall some basic concepts and properties from [3-5]:

Let $S$ be a set. A texturing $\mathcal{S}$ on $S$ is a subset of $\mathcal{P}(S)$ which is a point separating, complete, completely distributive lattice with respect to inclusion which contains $S, \emptyset$ and for which meet $\wedge$ coincides with intersection $\bigcap$ and finite joins $\bigvee$ with unions $\bigcup$. The pair $(S, \mathcal{S})$ is then called a texture or a texture space.

In general, a texturing of $S$ need not be closed under set complementation, but there may exist a mapping $\sigma: \mathcal{S} \rightarrow \mathcal{S}$ satisfying $\sigma(\sigma(A))=A$ and $A \subseteq B \Rightarrow \sigma(B) \subseteq \sigma(A)$ for all $A, B \in \mathcal{S}$. In this case $\sigma$ is called a complementation on $(S, \mathcal{S})$ and $(S, \mathcal{S}, \sigma)$ is said to be a complemented texture.

For a texture $(S, \mathcal{S})$, most properties are conveniently defined in terms of the $p-$ sets

$$
P_{s}=\bigcap\{A \in \mathcal{S} \mid s \in A\}
$$

and the $q-$ sets

$$
Q_{s}=\bigvee\{A \in \mathcal{S} \mid s \notin A\}=\bigvee\left\{P_{u} \mid u \in S, s \notin P_{u}\right\}
$$

A texture $(S, \mathcal{S})$ is called a plain texture if it satisfies any of the following equivalent conditions:

(1) $P_{s} \nsubseteq Q_{s}$ for all $s \in S$

(2) $A=\bigvee_{i \in I} A_{i}=\bigcup_{i \in I} A_{i}$ for all $A_{i} \in \mathcal{S}, i \in I$

For a set $A \in \mathcal{S}$, the core of $\mathrm{A}$ (denoted by $A^{\mathrm{b}}$ ) is defined by

$$
A^{b}=\bigcap\left\{\bigcup\left\{A_{i} \mid i \in I\right\} \mid\left\{A_{i} \mid i \in I\right\} \subseteq \mathcal{S}, A=\bigvee\left\{A_{i} \mid i \in I\right\}\right\} .
$$

Theorem 2.1 ([3]). In any texture space $(S, \mathcal{S})$, the following statements hold:

(1) $s \notin A \Rightarrow A \subseteq Q_{s} \Rightarrow s \notin A^{\text {b }}$ for all $s \in S, A \in \mathcal{S}$.

(2) $A^{\mathrm{b}}=\left\{s \mid A \nsubseteq Q_{s}\right\}$ for all $A \in \mathcal{S}$.

(3) For $A_{j} \in \mathcal{S}, j \in J$ we have $\left(\bigvee_{j \in J} A_{j}\right)^{b}=\bigcup_{j \in J} A_{j}^{b}$.

(4) $A$ is the smallest element of $\mathcal{S}$ containing $A^{b}$ for all $A \in \mathcal{S}$. 
(5) For $A, B \in \mathcal{S}$, if $A \nsubseteq B$ then there exists $s \in S$ with $A \nsubseteq Q_{s}$ and $P_{s} \nsubseteq \nsubseteq B$.

(6) $A=\bigcap\left\{Q_{s} \mid P_{s} \nsubseteq A\right\}$ for all $A \in \mathcal{S}$.

(7) $A=\bigvee\left\{P_{s} \mid A \nsubseteq Q_{s}\right\}$ for all $A \in \mathcal{S}$.

Example 2.1. (1) If $\mathcal{P}(X)$ is the powerset of a set $X$, then $(X, \mathcal{P}(X))$ is the discrete texture on $X$. For $x \in X, P_{x}=\{x\}$ and $Q_{x}=X \backslash\{x\}$. The mapping $\pi_{X}: \mathcal{P}(X) \rightarrow \mathcal{P}(X), \pi_{X}(Y)=X \backslash Y$ for $Y \subseteq X$ is a complementation on the texture $(X, \mathcal{P}(X))$.

(2) Setting $\mathbb{I}=[0,1], \mathcal{J}=\{[0, r),[0, r] \mid r \in \mathbb{I}\}$ gives the unit interval texture $(\mathbb{I}, \mathcal{J})$. For $r \in \mathbb{I}, P_{r}=[0, r]$ and $Q_{r}=[0, r)$. And the mapping $\iota: \mathcal{J} \rightarrow \mathcal{J}$, $\iota[0, r]=[0,1-r), \iota[0, r)=[0,1-r]$ is a complementation on this texture.

(3) The texture $(L, \mathcal{L}, \lambda)$ is defined by $L=(0,1], \mathcal{L}=\{(0, r] \mid r \in[0,1]\}$, $\lambda((0, r])=(0,1-r]$. For $r \in L, P_{r}=(0, r]=Q_{r}$.

(4) $\mathcal{S}=\{\emptyset,\{a, b\},\{b\},\{b, c\}, S\}$ is a simple texturing of $S=\{a, b, c\} . P_{a}=$ $\{a, b\}, P_{b}=\{b\}, P_{c}=\{b, c\}$. It is not possible to define a complementation on $(S, \mathcal{S})$.

(5) If $(S, \mathcal{S}),(V, \mathcal{V})$ are textures, the product texturing $\mathcal{S} \otimes \mathcal{V}$ of $S \times V$ consists of arbitrary intersections of sets of the form $(A \times V) \cup(S \times B)$, $A \in \mathcal{S}, B \in \mathcal{V}$, and $(S \times V, \mathcal{S} \otimes \mathcal{V})$ is called the product of $(S, \mathcal{S})$ and $(V, \mathcal{V})$. For $s \in S, v \in V, P_{(s, v)}=P_{s} \times P_{v}$ and $Q_{(s, v)}=\left(Q_{s} \times V\right) \cup\left(S \times Q_{v}\right)$.

A dichotomous topology, or ditopology for short, on a texture $(S, \mathcal{S})$ is a pair $(\tau, \kappa)$ of subsets of $\mathcal{S}$, where the set of open sets $\tau$ satisfies

$\left(T_{1}\right) S, \emptyset \in \tau$

$\left(T_{2}\right) G_{1}, G_{2} \in \tau \Rightarrow G_{1} \cap G_{2} \in \tau$

$\left(T_{3}\right) G_{i} \in \tau, i \in I \Rightarrow \bigvee_{i} G_{i} \in \tau$

and the set of closed sets $\kappa$ satisfies

$\left(C T_{1}\right) S, \emptyset \in \kappa$

$\left(C T_{2}\right) K_{1}, K_{2} \in \kappa \Rightarrow K_{1} \cup K_{2} \in \kappa$

$\left(C T_{3}\right) K_{i} \in \kappa, i \in I \Rightarrow \bigcap_{i} K_{i} \in \kappa$.

Hence a ditopology is essentially a "topology" for which there is no priori relation between the open and closed sets.

A complementation $\sigma$ on a texture $(S, \mathcal{S})$ is called grounded [13] if there is an involution $s \mapsto s^{\prime}$ on $S$ such that $\sigma\left(P_{s}\right)=Q_{s^{\prime}}$ and $\sigma\left(Q_{s}\right)=P_{s^{\prime}}\left(s^{\prime}\right.$ will be denoted by $\sigma(s))$ for all $s \in S$ and in this case the complemented texture space $(S, \mathcal{S}, \sigma)$ is called "complemented grounded texture space". In [16], it is shown that a complemented plain texture is grounded.

It is well known that, in the classical set theory, " $A \cap B=\emptyset$ if and only if $A \subseteq X \backslash B$ for any subsets, $A$ and $B$ of $X$ " however, in the fuzzy set theory, " $A \cap B=0$ implies $A \subset 1-B$ but in general, $A \subset 1-B$ doesn't imply $A \cap B=0$ for any fuzzy subsets, $A$ and $B$ of $X$ ". So it is defined an alternative binary implication in the fuzzy set theory such as; " $A$ is quasicoincident with $B$ (denoted by $A q B$ ) if and only if there exists an $x \in X$ 
such that $A(x)+B(x)>1$ for any fuzzy sets, $A$ and $B$ of $X$ " and " $A$ is not quasi-coincident with $B$ (denoted by $A \bar{q} B$ ) if and only if $A(x)+B(x) \leq 1$ for all $x \in X$ ". These notions are generalized to the complemented texture spaces as follows:

Let $(S, \mathcal{S}, \sigma)$ be a complemented texture space and $A, B \in \mathcal{S}$. It is called that " $A$ is quasi-coincident with $B$ " (denoted by $A q B$ ) if $A \nsubseteq \sigma(B)$ and " $A$ is not quasi-coincident with $B$ " (denoted by $A \bar{q} B$ ) if $A \subseteq \sigma(B)$ [11].

Definition $2.1([6])$. Let $(S, \mathcal{S}),(V, \mathcal{V})$ be textures and consider $\mathcal{T}, \mathcal{K}: \mathcal{S} \rightarrow$ $\mathcal{V}$ satisfying

$\left(G T_{1}\right) \mathcal{T}(S)=\mathcal{T}(\emptyset)=V$

$\left(G T_{2}\right) \mathcal{T}\left(A_{1}\right) \cap \mathcal{T}\left(A_{2}\right) \subseteq \mathcal{T}\left(A_{1} \cap A_{2}\right) \forall A_{1}, A_{2} \in \mathcal{S}$

$\left(G T_{3}\right) \bigcap_{j \in J} \mathcal{T}\left(A_{j}\right) \subseteq \mathcal{T}\left(\bigvee_{j \in J} A_{j}\right) \forall A_{j} \in \mathcal{S}, j \in J$

and

$\left(G C T_{1}\right) \mathcal{K}(S)=\mathcal{K}(\emptyset)=V$

$\left(G C T_{2}\right) \mathcal{K}\left(A_{1}\right) \cap \mathcal{K}\left(A_{2}\right) \subseteq \mathcal{K}\left(A_{1} \cup A_{2}\right) \forall A_{1}, A_{2} \in \mathcal{S}$

$\left(G C T_{3}\right) \bigcap_{j \in J} \mathcal{K}\left(A_{j}\right) \subseteq \mathcal{K}\left(\bigcap_{j \in J} A_{j}\right) \forall A_{j} \in \mathcal{S}, j \in J$

Then $\mathcal{T}$ is called a $(V, \mathcal{V})$-graded topology, $\mathcal{K}$ a $(V, \mathcal{V})$-graded cotopology and $(\mathcal{T}, \mathcal{K})$ a $(V, \mathcal{V})$-graded ditopology on $(S, \mathcal{S})$. The tuple $(S, \mathcal{S}, \mathcal{T}, \mathcal{K}, V, \mathcal{V})$ is called graded ditopological texture space.

For $v \in V$ it is defined that

$$
\mathcal{T}^{v}=\left\{A \in \mathcal{S} \mid P_{v} \subseteq \mathcal{T}(A)\right\}, \mathcal{K}^{v}=\left\{A \in \mathcal{S} \mid P_{v} \subseteq \mathcal{K}(A)\right\} .
$$

Then $\left(\mathcal{T}^{v}, \mathcal{K}^{v}\right)$ is a ditopology on $(S, \mathcal{S})$ for each $v \in V$.

That is, if $(S, \mathcal{S}, \mathcal{T}, \mathcal{K}, V, \mathcal{V})$ is any graded ditopological texture space, then there exists a ditopology $\left(\mathcal{T}^{v}, \mathcal{K}^{v}\right)$ on the texture space $(S, \mathcal{S})$ for each $v \in V$

If $(S, \mathcal{S}, \sigma)$ is a complemented texture and $(\mathcal{T}, \mathcal{K})$ a $(V, \mathcal{V})$-graded ditopology on $(S, \mathcal{S})$, then $(\mathcal{K} \circ \sigma, \mathcal{T} \circ \sigma)$ is also a $(V, \mathcal{V})$-graded ditopology on $(S, \mathcal{S})$. $(\mathcal{T}, \mathcal{K})$ is called complemented if $(\mathcal{T}, \mathcal{K})=(\mathcal{K} \circ \sigma, \mathcal{T} \circ \sigma)$.

Example 2.2. Let $(S, \mathcal{S}, \tau, \kappa)$ be a ditopological texture space and $(V, \mathcal{V})$ the discrete texture on a singleton. Take $(V, \mathcal{V})=(1, \mathcal{P}(1))$ (The notation 1 denotes the set $\{0\})$ and define $\tau^{g}: \mathcal{S} \rightarrow \mathcal{P}(1)$ by $\tau^{g}(A)=1 \Leftrightarrow A \in \tau$. Then $\tau^{g}$ is a $(V, \mathcal{V})$-graded topology on $(S, \mathcal{S})$. Likewise, $\kappa^{g}$ defined by $\kappa^{g}(A)=1 \Leftrightarrow A \in \kappa$ is a $(V, \mathcal{V})$-graded cotopology on $(S, \mathcal{S})$. $\left(\tau^{g}, \kappa^{g}\right)$ is called then the graded ditopology on $(S, \mathcal{S})$ corresponding to ditopology $(\tau, \kappa)$.

Therefore graded ditopological texture spaces are more general than ditopological texture spaces.

The graded Q-dineighborhood (Q-dinhd) systems of the graded ditopological texture spaces were defined in [8]. To avoid a long preliminaries we will give the following equivalent proposition instead of the definition. 
Proposition $2.1([8])$. Let $(\mathcal{T}, \mathcal{K})$ be a $(V, \mathcal{V})$-graded ditopology on $(S, \mathcal{S}, \sigma)$ and $\widetilde{N}, \widetilde{M}: S \rightarrow \mathcal{V}^{\mathcal{S}}$ mappings where $\widetilde{N}(s)=\widetilde{N}_{s}: \mathcal{S} \rightarrow \mathcal{V}, \widetilde{M}(s)=\widetilde{M}_{s}:$ $\mathcal{S} \rightarrow \mathcal{V}$ for each $s \in S$. Then $(\widetilde{N}, \widetilde{M})$ is a graded $Q$-dinhd system of the graded ditopological texture space $(S, \mathcal{S}, \mathcal{T}, \mathcal{K}, V, \mathcal{V})$ iff

$$
\widetilde{N}_{s}(A)= \begin{cases}\sup \left\{\mathcal{T}(B): P_{s} q B \subseteq A, B \in \mathcal{S}\right\}, & P_{s} q A \\ \emptyset, & P_{s} \bar{q} A\end{cases}
$$

$$
\widetilde{M}_{s}(A)= \begin{cases}\sup \left\{\mathcal{K}(B): P_{s} \nsubseteq B, A \subseteq B, B \in \mathcal{S}\right\}, & P_{s} \nsubseteq \subseteq \\ \emptyset, & P_{s} \subseteq A\end{cases}
$$

for each $s \in S$ and $A \in \mathcal{S}$.

Theorem $2.2([8])$. Let $(\mathcal{T}, \mathcal{K})$ be a $(V, \mathcal{V})$-graded ditopology on texture $(S, \mathcal{S})$. If $(\widetilde{N}, \widetilde{M})$ is the graded $Q$-dinhd system of graded ditopological texture space $(S, \mathcal{S}, \mathcal{T}, \mathcal{K}, V, \mathcal{V})$, then the following properties hold for each $s \in S$, $A, A_{1}, A_{2} \in \mathcal{S}:$

(1) $(\tilde{\mathbf{N}} 1) \widetilde{N}_{s}(A) \neq \emptyset \Rightarrow P_{s} q A$

$(\tilde{\mathbf{N}} 2) \tilde{N}_{s}(\emptyset)=\emptyset$ and $\widetilde{N}_{s}(S)=V$

$(\tilde{\mathbf{N}} 3) A_{1} \subseteq A_{2} \Rightarrow \widetilde{N}_{s}\left(A_{1}\right) \subseteq \widetilde{N}_{s}\left(A_{2}\right)$

$(\widetilde{\mathbf{N}} 4) \widetilde{N}_{s}\left(A_{1}\right) \wedge \widetilde{N}_{s}\left(A_{2}\right) \subseteq \widetilde{N}_{s}\left(A_{1} \cap A_{2}\right)$

( $\mathbf{N} 5) \tilde{N}_{s}(A)=\sup \left\{\bigwedge_{P_{s^{\prime}} q B} \widetilde{N}_{s^{\prime}}(B): P_{s} q B \subseteq A\right\}$

(2) $(\widetilde{\mathrm{M}} 1) \widetilde{M}_{s}(A) \neq \emptyset \Rightarrow P_{s} \nsubseteq A$

$(\widetilde{\mathbf{M}} 2) \widetilde{M}_{s}(S)=\emptyset$ and $\widetilde{M}_{s}(\emptyset)=V$

$(\widetilde{\mathrm{M}} 3) A_{1} \subseteq A_{2} \Rightarrow \widetilde{M}_{s}\left(A_{2}\right) \subseteq \widetilde{M}_{s}\left(A_{1}\right)$

$(\widetilde{\mathbf{M}} 4) \widetilde{M}_{s}\left(A_{1}\right) \wedge \widetilde{M}_{s}\left(A_{2}\right) \subseteq \widetilde{M}_{s}\left(A_{1} \cup A_{2}\right)$

$(\widetilde{\mathbf{M}} 5) \widetilde{M}_{s}(A)=\sup \left\{\bigwedge_{P_{s^{\prime}} \nsubseteq B} \widetilde{M}_{s^{\prime}}(B): P_{s} \nsubseteq B, A \subseteq B\right\}$

Definition $2.2([9])$. Let $(S, \mathcal{S})$ and $(V, \mathcal{V})$ be textures.

(1) A mapping $\mathfrak{F}: \mathcal{S} \rightarrow \mathcal{V}$ is called a $(V, \mathcal{V})$-graded filter on $(S, \mathcal{S})$ if $\mathfrak{F}$ satisfies:

(GF1) $\mathfrak{F}(\emptyset)=\emptyset$

(GF2) $A_{1} \subseteq A_{2} \Rightarrow \mathfrak{F}\left(A_{1}\right) \subseteq \mathfrak{F}\left(A_{2}\right)$

(GF3) $\mathfrak{F}\left(A_{1}\right) \wedge \mathfrak{F}\left(A_{2}\right) \subseteq \mathfrak{F}\left(A_{1} \cap A_{2}\right)$

(2) A mapping $\mathfrak{G}: \mathcal{S} \rightarrow \mathcal{V}$ is called a $(V, \mathcal{V})$-graded cofilter on $(S, \mathcal{S})$ if $\mathfrak{G}$ satisfies:

(GCF1) $\mathfrak{G}(S)=\emptyset$

$(\mathrm{GCF} 2) \quad A_{1} \subseteq A_{2} \Rightarrow \mathfrak{G}\left(A_{2}\right) \subseteq \mathfrak{G}\left(A_{1}\right)$

$(\mathrm{GCF} 3) \mathfrak{G}\left(A_{1}\right) \wedge \mathfrak{G}\left(A_{2}\right) \subseteq \mathfrak{G}\left(A_{1} \cup A_{2}\right)$

(3) If $\mathfrak{F}$ is a $(V, \mathcal{V})$-graded filter and $\mathfrak{G}(V, \mathcal{V})$-graded cofilter on $(S, \mathcal{S})$ then the pair $(\mathfrak{F}, \mathfrak{G})$ is called a $(V, \mathcal{V})$-graded difilter on $(S, \mathcal{S})$. 
Definition $2.3([9])$. $(V, \mathcal{V})$-graded difilter $(\mathfrak{F}, \mathfrak{G})$ on $(S, \mathcal{S})$ is called regular if it satisfies the following equivalent conditions:

(1) $\mathfrak{F} \wedge \mathfrak{G}=\emptyset$ i.e. $\mathfrak{F}(A) \wedge \mathfrak{G}(A)=\emptyset$ for all $A \in \mathcal{S}$.

(2) $\forall n \in \mathbb{N}, \bigwedge_{i=1}^{n}\left(\mathfrak{F}\left(A_{i}\right) \wedge \mathfrak{G}\left(B_{i}\right)\right) \neq \emptyset \Rightarrow \bigcap_{i=1}^{n} A_{i} \nsubseteq \bigcup_{i=1}^{n} B_{i}$, for all $A_{i}, B_{i} \in \mathcal{S}$

(3) $\mathfrak{F}(A) \wedge \mathfrak{G}(B) \neq \emptyset \Rightarrow A \nsubseteq B$, for all $A, B \in \mathcal{S}$

Definition $2.4([9])$. et $(S, \mathcal{S}, \mathcal{T}, \mathcal{K}, V, \mathcal{V})$ be a graded ditopological texture space, $A \in \mathcal{S}$ and $v \in V$. The set

$$
\bigcap\left\{B \in \mathcal{S} \mid A \subseteq B, P_{v} \subseteq \mathcal{K}(B)\right\} \in \mathcal{S}
$$

is called v-closure of $A$ and denoted by $[A]^{v}$. The set

$$
\bigvee\left\{B \in \mathcal{S} \mid B \subseteq A, P_{v} \subseteq \mathcal{T}(B)\right\} \in \mathcal{S}
$$

is called v-interior of $A$ and denoted by $] A{ }^{v}$.

Note that for each $v \in V,[A]^{v}(] A\left[^{v}\right)$ is closure (interior) of the set $A$ in ditopological texture space $\left(S, \mathcal{S}, \mathcal{T}^{v}, \mathcal{K}^{v}\right)$.

Definition $2.5([9])$. et $(\mathfrak{F}, \mathfrak{G})$ be a $(V, \mathcal{V})$-graded difilter on $(S, \mathcal{S})$. $(\mathfrak{F}, \mathfrak{G})$ is called a maximal $(V, \mathcal{V})$-graded difilter on $(S, \mathcal{S})$ if whenever $\left(\mathfrak{F}^{\prime}, \mathfrak{G}^{\prime}\right)$ is a $(V, \mathcal{V})$-graded difilter on $(S, \mathcal{S})$ and $(\mathfrak{F}, \mathfrak{G}) \subseteq\left(\mathfrak{F}^{\prime}, \mathfrak{G}^{\prime}\right)$ then we have $(\mathfrak{F}, \mathfrak{G})=$ $\left(\mathfrak{F}^{\prime}, \mathfrak{G}^{\prime}\right)$ is hold.

\section{Main Results}

From now on, we assume that $(S, \mathcal{S}, \sigma)$ is a complemented grounded texture space.

Example 3.1. Let $(S, \mathcal{S}, \mathcal{T}, \mathcal{K}, V, \mathcal{V})$ be a graded ditopological texture space and $(\widetilde{N}, \widetilde{M})$ the graded Q-dinhd system of $(S, \mathcal{S}, \mathcal{T}, \mathcal{K}, V, \mathcal{V})$. Then for each $s \in S, \widetilde{N}_{s}$ is a $(V, \mathcal{V})$-graded filter and $\widetilde{M}_{s}$ is a $(V, \mathcal{V})$-graded cofilter on $(S, \mathcal{S})$. Moreover $\left(\widetilde{N}_{s}, \widetilde{M}_{\sigma(s)}\right)$ is a regular $(V, \mathcal{V})$-graded difilter on $(S, \mathcal{S})$.

Indeed; (GF1): We have $\widetilde{N}_{s}(\emptyset)=\emptyset$ by $(\widetilde{N} 2)$. (GF2): $A_{1} \subseteq A_{2} \Rightarrow$ $\widetilde{N}_{s}\left(A_{1}\right) \subseteq \widetilde{N}_{s}\left(A_{2}\right)$ by $(\widetilde{N} 3) .(\mathrm{GF} 3): \widetilde{N}_{s}\left(A_{1}\right) \wedge \widetilde{N}_{s}\left(A_{2}\right) \subseteq \widetilde{N}_{s}\left(A_{1} \cap A_{2}\right)$ by $(\widetilde{N} 4)$. So, $\widetilde{N}_{s}$ is a $(V, \mathcal{V})$-graded filter on $(S, \mathcal{S})$ for each $s \in S$. Similarly it can be shown that $\widetilde{M}_{s}$ is a $(V, \mathcal{V})$-graded cofilter on $(S, \mathcal{S})$ for each $s \in S$.

For a set $A \in \mathcal{S}, P_{s} q A$ or $P_{s} \bar{q} A$. If $P_{s} q A$ then we have $P_{s} \nsubseteq \sigma(A)$ and so $A \nsubseteq Q_{\sigma(s)}$. Thus we get $P_{\sigma(s)} \subseteq A$ and so $\widetilde{M}_{\sigma(s)}(A)=\emptyset$. If $P_{s} \bar{q} A$ then we have $\widetilde{N}_{s}(A)=\emptyset$. Therefore we have $\widetilde{N}_{s}(A) \wedge \widetilde{M}_{\sigma(s)}(A)=\emptyset$ and so $\left(\widetilde{N}_{s}, \widetilde{M}_{\sigma(s)}\right)$ is a regular $(V, \mathcal{V})$-graded difilter on $(S, \mathcal{S})$.

Definition 3.1. Let $(\mathfrak{F}, \mathfrak{G})$ be a $(V, \mathcal{V})$-graded difilter on $(S, \mathcal{S})$. We say that $\mathfrak{F}$ Q-converges to $s$ and write that $\mathfrak{F} \longrightarrow^{q} s$ if $\widetilde{N}_{s} \subseteq \mathfrak{F}$. Again, we say that $\mathfrak{G}$ Q-converges to $s$ and write that $\mathfrak{G} \longrightarrow^{q} s$ if $\widetilde{M}_{s} \subseteq \mathfrak{G}$. 
For $s \in S$, the graded difilter $(\mathfrak{F}, \mathfrak{G})$ is called Q-diconvergent if $\mathfrak{F} \longrightarrow^{q} s$ and $\mathfrak{G} \longrightarrow^{q} \sigma(s)$. In this case, the couple $(s, \sigma(s))$ or $s$ for short is called Q-limit of $(\mathfrak{F}, \mathfrak{G})$.

Proposition 3.1. For a graded difilter $(\mathfrak{F}, \mathfrak{G})$ on $(S, \mathcal{S}, \mathcal{T}, \mathcal{K}, V, \mathcal{V})$ the following hold:

(a) $\mathfrak{F} \longrightarrow$ " $s \Leftrightarrow$ "P $P_{s} q A \Rightarrow \mathcal{T}(A) \subseteq \mathfrak{F}(A)$ "

(b) $\mathfrak{G} \longrightarrow^{q} s \Leftrightarrow$ "P $P_{s} \nsubseteq A \Rightarrow \mathcal{K}(A) \subseteq \mathfrak{G}(A)$ ".

Proof. (a) $(\Rightarrow)$ : Let $\mathfrak{F} \longrightarrow^{q} s$ and $P_{s} q A$. Since $\mathfrak{F} \longrightarrow q$ we have $\widetilde{N}_{s} \subseteq \mathfrak{F}$ and so $\widetilde{N}_{s}(A) \subseteq \mathfrak{F}(A)$. On the other hand, $P_{s} q A$ implies $T(A) \subseteq \widetilde{N}_{s}(A)$. Hence $\mathcal{T}(A) \subseteq \mathfrak{F}(A)$ is obtained.

$(\Leftarrow)$ : Considering the definition of $\widetilde{N}_{s}$, we get $\widetilde{N}_{s}(A) \subseteq \mathfrak{F}(A)$ because of the implication $P_{s} q A \Rightarrow \mathcal{T}(A) \subseteq \mathfrak{F}(A)$. Thus we have $\mathfrak{F} \longrightarrow^{q} s$.

The proof of (b) is similar to (a).

Proposition 3.2. For a regular graded difilter $(\mathfrak{F}, \mathfrak{G})$ on $(S, \mathcal{S}, \mathcal{T}, \mathcal{K}, V, \mathcal{V})$ the following hold:

(a) $\left.\mathfrak{F} \longrightarrow^{q} s \Rightarrow " \forall A \in \mathcal{S}, v \in \mathfrak{G}(A) \Rightarrow P_{s} \bar{q}\right] A[v "$

(b) $\mathfrak{G} \longrightarrow q$ " $s \Rightarrow \forall A \in \mathcal{S}, v \in \mathfrak{F}(A) \Rightarrow P_{s} \subseteq[A]^{v "}$.

Proof. (a) Let $\mathfrak{F} \longrightarrow^{q} s$ and suppose that there exists $v \in \mathfrak{G}(A)$ such that $\left.P_{s} q\right] A{ }^{v}$ for a set $A \in \mathcal{S}$. Since $\left.P_{s} q\right] A{ }^{v}$ we have $P_{s} \nsubseteq \sigma(\bigvee\{B \in \mathcal{S} \mid B \subseteq$ $\left.\left.A, P_{v} \subseteq \mathcal{T}(B)\right\}\right)=\bigcap\left\{\sigma(B) \mid B \subseteq A, P_{v} \subseteq \mathcal{T}(B)\right\}$ and so, there exists $B \in \mathcal{S}$ such that $P_{s} \nsubseteq \sigma(B), B \subseteq A, P_{v} \subseteq \mathcal{T}(B)$. Because $\mathfrak{F} \longrightarrow^{q} s$ and $P_{s} q B$, using Proposition 3.1 we have $\mathcal{T}(B) \subseteq \mathfrak{F}(B)$. So we get $P_{v} \subseteq \mathcal{T}(B) \subseteq$ $\mathfrak{F}(B) \subseteq \mathfrak{F}(A)$ and remembering $v \in \mathfrak{G}(A)$ we obtain $\mathfrak{F}(A) \cap \mathfrak{G}(A) \neq \emptyset$ which contradicts with the regularity of $(\mathfrak{F}, \mathfrak{G})$. The proof of $(b)$ is similar to (a).

Definition 3.2. Let $(\mathfrak{F}, \mathfrak{G})$ be a regular $(V, \mathcal{V})$-graded difilter on a graded ditopological texture space $(S, \mathcal{S}, \mathcal{T}, \mathcal{K}, V, \mathcal{V})$.

(1) $s \in S$ is called a Q-cluster point of $\mathfrak{G}$ if " $\left.v \in \mathfrak{G}(A) \Rightarrow P_{s} \bar{q}\right] A[v$ " for all $A \in \mathcal{S}$.

(2) $s \in S$ is called a Q-cluster point of $\mathfrak{F}$ if " $v \in \mathfrak{F}(A) \Rightarrow P_{s} \subseteq[A]$ " for all $A \in \mathcal{S}$.

(3) For $s \in S$ if $s$ is a Q-cluster point of $\mathfrak{F}$ and $\sigma(s)$ is a Q-cluster point of $\mathfrak{G}$ then $(\mathfrak{F}, \mathfrak{G})$ is called Q-diclustering in $(S, \mathcal{S}, \mathcal{T}, \mathcal{K}, V, \mathcal{V})$.

Corollary 3.1. In a graded ditopological texture space, each Q-diconvergent difilter is Q-diclustering.

Theorem $3.1([9])$. If $(\mathfrak{F}, \mathfrak{G})$ is a regular $(V, \mathcal{V})$-graded difilter on $(S, \mathcal{S})$ and $(V, \mathcal{V})$ is discrete, then the following are equivalent:

(1) $(\mathfrak{F}, \mathfrak{G})$ is a maximal regular $(V, \mathcal{V})$-graded difilter.

(2) $\mathfrak{F} \vee \mathfrak{G}=V$ (i.e. $\forall A \in \mathcal{S}, \mathfrak{F}(A) \vee \mathfrak{G}(A)=\mathfrak{F}(A) \cup \mathfrak{G}(A)=V$ ). 
Theorem 3.2. Let $(V, \mathcal{V})$ be a discrete texture space and $(\mathfrak{F}, \mathfrak{G})$ a maximal regular graded difilter in $(S, \mathcal{S}, \mathcal{T}, \mathcal{K}, V, \mathcal{V})$ then $(\mathfrak{F}, \mathfrak{G})$ is $Q$-diconvergent if and only if it is $Q$-diclustering.

Proof. If $(\mathfrak{F}, \mathfrak{G})$ is Q-diconvergent then it is Q-diclustering by Corollary 3.1. On the other part, let $(\mathfrak{F}, \mathfrak{G})$ be Q-diclustering. Particularly let $s$ be a $\mathrm{Q}$ cluster point of $\mathfrak{F}$ and $\sigma(s)$ a Q-cluster point of $\mathfrak{G}$. Then $\left.v \in \mathfrak{G}(A) \Rightarrow P_{s} \bar{q}\right] A\left[^{v}\right.$ and $v \in \mathfrak{F}(A) \Rightarrow P_{s} \subseteq[A]^{v}$ for all $A \in \mathcal{S}$.

Let $A \in \mathcal{S}$ with $A \nsubseteq Q_{\sigma(s)}$ and $v \notin \mathfrak{F}(A)$. Since $(\mathfrak{F}, \mathfrak{G})$ is maximal we have $\mathfrak{F}(A) \cup \mathfrak{G}(A)=V$ by Theorem 3.1. So, we get $v \in \mathfrak{G}(A)$ because of the regularity of $(\mathfrak{F}, \mathfrak{G})$ and $\left.P_{s} \bar{q}\right] A\left[{ }^{v}\right.$. If we suppose that $v \in \mathcal{T}(A)$ then we have $\left.P_{s} \bar{q}\right] A\left[{ }^{v}=A\right.$ and so $A \subseteq Q_{\sigma(s)}$ which contradicts with $A \nsubseteq Q_{\sigma(s)}$. Hence we get $v \notin \mathcal{T}(A)$. Therefore we have the implication $v \notin \mathfrak{F}(A) \Rightarrow v \notin \mathcal{T}(A)$ i.e. $\mathcal{T}(A) \subseteq \mathfrak{F}(A)$. Since $P_{s} q A$ implies $A \nsubseteq Q_{\sigma(s)}$ we have $\mathfrak{F} \longrightarrow^{q} s$ by Proposition 3.1. It can be also obtained that $\mathfrak{G} \longrightarrow^{q} \sigma(s)$ by means of the similar method. Thus $(\mathfrak{F}, \mathfrak{G})$ is Q-diconvergent.

Proposition $3.3([9])$. Let $(\mathfrak{F}, \mathfrak{G})$ be a regular $(V, \mathcal{V})$-graded difilter on $(S, \mathcal{S})$. Then there exists a maximal regular $(V, \mathcal{V})$-graded difilter $\left(\mathfrak{F}^{M}, \mathfrak{G}^{M}\right)$ on $(S, \mathcal{S})$ such that $(\mathfrak{F}, \mathfrak{G}) \subseteq\left(\mathfrak{F}^{M}, \mathfrak{G}^{M}\right)$.

Theorem 3.3. Let $(S, \mathcal{S}, \mathcal{T}, \mathcal{K}, V, \mathcal{V})$ be a graded ditopological texture space. Then for the statements:

(a) every regular graded difilter on $(S, \mathcal{S}, \mathcal{T}, \mathcal{K}, V, \mathcal{V})$ is $Q$-diclustering,

(b) every maximal regular graded difilter on $(S, \mathcal{S}, \mathcal{T}, \mathcal{K}, V, \mathcal{V})$ is Q-diconvergent,

the implication $(b) \Rightarrow(a)$ and in case of $(V, \mathcal{V})$ is discrete, $(a) \Rightarrow(b)$ hold.

Proof. $(a) \Rightarrow(b)$ : Let $(\mathfrak{F}, \mathfrak{G})$ be a maximal regular graded difilter on $(S, \mathcal{S}, \mathcal{T}, \mathcal{K}, V, \mathcal{V})$. Because of $(\mathrm{a}),(\mathfrak{F}, \mathfrak{G})$ is Q-diclustering. Since $(V, \mathcal{V})$ is discrete, we obtain that $(\mathfrak{F}, \mathfrak{G})$ is Q-diconvergent by Theorem 3.2.

$(b) \Rightarrow(a)$ : Let $(\mathfrak{F}, \mathfrak{G})$ be a regular graded difilter on $(S, \mathcal{S}, \mathcal{T}, \mathcal{K}, V, \mathcal{V})$. Then there exists a maximal regular graded difilter $\left(\mathfrak{F}^{M}, \mathfrak{G}^{M}\right)$ on $(S, \mathcal{S}, \mathcal{T}, \mathcal{K}, V, \mathcal{V})$ with $(\mathfrak{F}, \mathfrak{G}) \subseteq\left(\mathfrak{F}^{M}, \mathfrak{G}^{M}\right)$ by Proposition 3.3. Using (b) we have $\mathfrak{F}^{M} \longrightarrow^{q} s$ and $\mathfrak{G}^{M} \longrightarrow^{q} \sigma(s)$ for some $s \in S$. Since $\mathfrak{G}^{M} \longrightarrow^{q} \sigma(s)$, using Proposition 3.2 we get $v \in \mathfrak{F}(A) \Rightarrow v \in \mathfrak{F}^{M}(A) \Rightarrow P_{\sigma(s)} \subseteq[A]^{v}$ for each $A \in \mathcal{S}$. So, $\sigma(s)$ is a Q-cluster point of $\mathfrak{F}$. Similarly one can get that $s$ is a Q-cluster point of $\mathfrak{G}$. Considering $\sigma(\sigma(s))=s$, we get that $(\mathfrak{F}, \mathfrak{G})$ is Q-diclustering.

\section{Conclusion}

Convergence of filter is very useful in topological spaces as it describes several topological concepts. In this paper, using graded Q-dinhds, an alternative definition of convergence of graded difilters has been introduced. This new convergence type (i.e. Q-convergence) has some advantages and 
some disadvantages in comparison with the convergence defined in [9]. Convergence of graded difilters is not defined using graded dineighborhoods but a version of it because in general, a graded nhd is not a graded difilter. Contrary to the concept of convergence, Q-convergence is defined directly by means of Q-dinhds since $\left(\widetilde{N}_{s}, \widetilde{M}_{\sigma(s)}\right)$ is a regular graded difilter (see Example 3.1). However, there is a handicap of this new structure: Q-convergence is defined on complemented grounded textures.

\section{REFERENCES}

[1] L. M. Brown and M. Diker, Ditopological texture spaces and intuitionistic sets, Fuzzy Sets and Systems, 98 (1998), 217-224.

[2] L. M. Brown and R. Ertürk, Fuzzy sets as texture spaces, I. Representation theorems, Fuzzy Sets and Systems, 110(2) (2000), 227-236.

[3] L. M. Brown, R. Ertürk, Ş. Dost, Ditopological texture spaces and fuzzy topology, I. Basic concepts, Fuzzy Sets and Systems, 147(2) (2004), 171-199.

[4] L. M. Brown, R. Ertürk, Ş. Dost, Ditopological texture spaces and fuzzy topology, II. Topological considerations, Fuzzy Sets and Systems, 147(2) (2004), 201-231.

[5] L. M. Brown, R. Ertürk, Ş. Dost, Ditopological texture spaces and fuzzy topology, III. Separation Axioms, Fuzzy Sets and Systems, 157(14) (2006), 1886-1912.

[6] L. M. Brown, A. Šostak, Categories of fuzzy topology in the context of graded ditopologies on textures, Iranian Journal of Fuzzy Systems, 11:6 (2014) 1-20.

[7] C. L. Chang, Fuzzy topological spaces, Journal of Mathematical Analysis and Applications, 24 (1968) 182-190.

[8] R. Ekmekçi and R. Ertürk, Neighborhood structures of graded ditopological texture spaces, Filomat, 29:7 (2015), 1445-1459.

[9] R. Ekmekçi and R. Ertürk, Convergence in graded ditopological texture spaces, Applied General Topology, 17:1 (2016), 17-35.

[10] R. Ertürk, Seperation axioms in fuzzy topology characterized by bitopologies, Fuzzy Sets and Systems, 58 (1993), 206-209.

[11] R. Ertürk, Ş. Dost, S. Özçă̆, Generalization some fuzzy separation axioms to ditopological texture spaces, The Journal of Nonlinear Sciences and Applications, 2 (2009), 234-242.

[12] T. Kubiak, On fuzzy topologies, Ph.D. Thesis, A. Mickiewicz University Poznan, Poland, 1985. 
[13] S. Özçă̆ and L. M. Brown, The prime dicompletion of a di-uniformity on a plain texture, Topology and its Applications, 158 (2011), 15841594.

[14] A. Šostak, On a fuzzy topological structure, Rendiconti Circolo Matematico Palermo, Serie II No. 11 (1985) 89-103.

[15] A. Šostak, Two decates of fuzzy topology: basic ideas, notions and results, Russian Math. Surveys, 44:6 (1989) 125-186.

[16] İ. U. Tiryaki, L. M. Brown, Plain ditopological texture spaces, Topology and its Applications 158 (2011) 2005-2015.

[17] F. Yıldız and S. Özçağ, The ditopology generated by pre-open and preclosed sets, and submaximality in textures, Filomat, 27:1 (2013) 95-107.

[18] F. Yıldız, Connections between realcompactifications in various categories, Quaestiones Mathematicae, 38:3 (2015) 431-455.

\section{RAMAzAN EKMEKÇi}

Department of Mathematics

Çanakkale Onsekiz Mart University

17020, ÇANAKKaLE

TURKEY

E-mail address: ekmekci@comu.edu.tr

\section{RIZA ERTÜRK}

Department of Mathematics

HACETTEPE UNIVERSITY

06800, ANKARA,

TURKEY

E-mail address: rerturk@hacettepe.edu.tr 